\author{
Fabien S. Godeferd ${ }^{1}$ \\ LMFA UMR 5509 CNRS, \\ École centrale de Lyon, \\ Université de Lyon, \\ Écully 69134, France \\ e-mail: fabien.godeferd@ec-lyon.fr \\ Frédéric Moisy \\ FAST UMR 7608 CNRS, \\ Université Paris-Sud, \\ Orsay 91405, France \\ e-mail: frederic.moisy@fast.u-psud.fr
}

\section{Structure and Dynamics of Rotating Turbulence: A Review of Recent Experimental and Numerical Results}

\begin{abstract}
Rotating turbulence is a fundamental phenomenon appearing in several geophysical and industrial applications. Its study benefited from major advances in the recent years, but also raised new questions. We review recent results for rotating turbulence, from several numerical and experimental researches, and in relation with theory and models, mostly for homogeneous flows. We observe a convergence in the statistical description of rotating turbulence from the advent of modern experimental techniques and computational power that allows to investigate the structure and dynamics of rotating flows at similar parameters and with similar description levels. The improved picture about the anisotropization mechanisms, however, reveals subtle differences in the flow conditions, including its generation and boundary conditions, which lead to separate points of view about the role of linear mechanisms - the Coriolis force and inertial waves-compared with more complex nonlinear triadic interactions. This is discussed in relation with the most recent diagnostic of dynamical equations in physical and spectral space.
\end{abstract} [DOI: $10.1115 / 1.4029006]$

\section{Introduction}

Among a wide range of distortions that can affect the properties of turbulent flows, rotation is widely studied from its presence in several contexts, from astrophysical and geophysical to industrial flows. On Earth or on planets with atmospheres as Jupiter, the scales are such that the flows are essentially turbulent, and the Coriolis force induced by rotation about the polar axis is responsible for a complex dynamics without which strong coherent structures would not exist: instances are cyclones on the Earth or the great red spot in the Jovian atmosphere. Various rotation rates may be encountered now that several extrasolar planets have been found (the variability of rotation rates in their host stars is reported, e.g., in Ref. [1]), and this justifies parametric investigations that help to link orbital disturbances to the internal behavior of planetary flows, partly driven by solid body rotation. In giant planets such as Jupiter, the atmospheric circulation is strongly affected by rotation, in addition to density effects, with variable intensities found in extrasolar planets [2]. Back on Earth, the atmosphere has a dynamics strongly related to the diurnal cycle, which is also the case of flows in the ocean, although the scales involved in atmospheric flow dynamics may be different from the oceanic ones.

At human level, rotation also modifies the flow dynamics through the Coriolis force. This is encountered in turbomachinery, especially in large-scale energy producing water or wind turbines [3], and in compressors or turbines of turbofan reactors where very large rotation rates are present, up to $10^{5} \mathrm{rpm}$. In these interblade flows, however, pressure and compressibility effects may be dominant over rotation ones. In industrial flows, rotation is therefore also in competition with finite-size effects and the presence of other distortions which may be of large amplitude; for instance, walls or solid bodies introduce velocity gradients that often redistribute energy more efficiently, or faster, than Coriolis force

${ }^{1}$ Corresponding author.

Manuscript received January 10, 2014; final manuscript received October 30 , 2014; published online January 15, 2015. Assoc. Editor: Herman J. H. Clercx. does. Density variations can also often be strong, and introduce buoyancy forces that interact with the Coriolis force [4]. There lies an important specificity of rotating flows, in that the Coriolis force does no work, unlike other kinds of body forces, Lorentz, buoyancy, etc., and is not a production term in the same sense as velocity-gradient-related production in the energy balance equation [5]. Rotation, however, does redistribute energy in its own specific way, which is not the object of a wide consensus, concerning its modeling. This focus particularly questions the linear or nonlinear nature of the effect of rotation on turbulent flows, and a univocal answer is not yet available, since an accurate analysis of the different timescales and topological effects involved in the dynamics is required, all effects that may drive rotating flows to different regimes. Among the parameters to account for this complexity, the flow regime is utterly important, characterized by the Rossby number $\operatorname{Ro}=U /(2 \Omega \ell)$ and the Reynolds number $\operatorname{Re}=U \ell / \nu-U$ is a velocity scale associated with an integral length scale of turbulence $\ell$. One may also list other effects, such as confinement, slight asymmetries or perturbations-e.g., biases induced by periodic boundary conditions in simulations - and the initial conditions.

In the presence of solid walls, rotation can become a production term, and different kinds of boundary layers appear depending on the orientation of the wall: Ekman layers develop along horizontal boundaries when the orientation of rotation is vertical-which we are going to assume from now on, with $z$ the vertical directionwhereas Stewartson layers develop on vertical boundaries [6,7]. The Ekman number $E=\nu /\left(2 \Omega L^{2}\right)$ —rotation rate $\Omega$, container size $L$, kinematic viscosity $\nu$-is used to characterize the thickness of these layers, being for instance $L E^{1 / 2}$ for the Ekman layers. We shall focus in this review on the dynamics of rotating turbulence away from solid boundaries, assuming negligible transport phenomena linked with the presence of remote walls, such as Ekman pumping. In addition, the homogeneity assumption is valid only if the integral scale $\ell$ is significantly smaller than the size of the fluid domain $L$.

The dynamics of homogeneous rotating turbulence is therefore a rather intricate issue, which has been tackled in different ways, 
with models ranging from simple to sophisticated, starting from linear approximations and extending to full nonlinear modeling of third-order velocity statistics. The famed Taylor-Proudman theorem applies to rapidly rotating flows $[6,8,9]$ : whenever forcing imposes an external timescale, the neglect of velocity derivatives along the rotation axis is permitted. Fluid columns are therefore set in motion like solid bodies, and the flow may be considered to be two-dimensional, although the vertical velocity component not necessarily vanishes, and can behave as a convected scalar. The degree to which homogeneous rotating turbulence becomes naturally two-dimensional is debated and, as mentioned, depends on several factors. Considering freely decaying turbulence initiated with given conditions, the Taylor-Proudman theorem does not apply to explain the two-dimensionalization of the flow, which also strongly depends on the value of the Rossby number Ro. When Ro $\gg 1$, the turbulent flow evolves much faster than the timescale over which rotation can be felt, and is thus unperturbed by rotation. In statistically steady rotating turbulence, a lowenough Rossby number is required for rotation to affect significantly the flow dynamics. In freely decaying turbulence, or in spatially evolving turbulent flows-e.g., in wind tunnel experiments, or the spatial diffusion of a turbulent patch - the Rossby number is required to decrease to values below unity for rotation to effectively modify the flow dynamics. At low values, Ro $<0.1$ or below, the rotation timescale $1 / \Omega$ is small enough for the Coriolis force to have time to change a turbulent structure which evolves over a timescale $\ell / U$, and it progressively affects smaller scales when the Rossby number decreases. Thus, considering very large Reynolds number Re, or infinite, the asymptotic limit Ro $\rightarrow 0$ is an interesting one, considered mathematically in Ref. [10]. For more realistic values, at finite Reynolds number, the Coriolis force affects differently large and small scales. The comparison of the Coriolis timescale to that of turbulent structures of a given size led Zeman [11] to introduce the scale $l_{\Omega}$ at which the inertial and eddy turnover times are equal. The Zeman scale may therefore be considered as a demarcation value above which large scales are affected by rotation while the small scales below $l_{\Omega}$ may recover isotropic turbulence properties.

Different regimes are illustrated as a function of Rossby and Reynolds number in the schematic of Fig. 1. Inertial waves are observed in the linear, low Reynolds number regime, whereas weak interactions are expected to occur in the wave turbulence regime encountered asymptotically at vanishing Rossby number for high Reynolds number flows. Quasi-two-dimensional turbulence can be observed for sufficiently energetic flows at Ro $<1$.

Not only does rotation act differentially on the various scales of turbulence, but the Coriolis force modifies the turbulent dynamics and therefore the turbulent structures in a different way along the rotation axis or in perpendicular planes. The above-mentioned trend toward two-dimensionality is a manifestation of the anisotropic pressure effect which couples horizontal and vertical velocity components. As a result, thanks to the presence of the Coriolis force which bends fluid particles' trajectories, inertial wave propagation is permitted, with a dispersion relation for the time frequency $\sigma=2 \Omega \cos \theta$, where $\theta$ is the angle between the wavevector and the normal to the rotation axis [12]. Inertial waves are plane waves that follow the linearized momentum equations set in a rotating frame. Therefore, rotating turbulence may be modeled as a superposition of inertial waves propagating from perturbations that act as sources within the flow, and thus be amenable to wave-turbulence theory, or, in a more intensely driven regime, as strongly nonlinear interactions between motions which are constrained by rotation. In the latter case, inertial waves may not be observed over long propagation ranges, but models based on their nonlinear interactions still appear to be relevant.

Recent significant advances have been made to further understand and model the dynamics and structuration of rotating turbulence; for instance, devoted high resolution direct numerical simulations and state-of-the-art experiments have permitted to describe the precise anisotropic energy contents of rotating turbulence both in spectral and physical statistical formalisms [13,14]. However, the exhaustive modeling of rotating turbulence at all possible regimes is far from being reached, and further efforts are required.

Our review concerns recent advances about rotating turbulence, and complements presentations found in recent textbooks $[5,15]$. After discussing the properties of inertial waves and of the dynamical equations, we shall inspect in the following the abovementioned concepts developed in several approaches of rotating turbulence, focusing on geometries in which wall effects are expected to be small, in experiments (Sec. 3), or in numerical simulations with periodic boundary conditions (Sec. 4). We will then mention how the anisotropic description of rotating turbulence is approached in various statistical characterizations, and how the dynamics of rotating turbulence is still a complex debated subject.

\section{Inertial Waves and Wave Turbulence in Rotating Flows}

Rotating turbulence may be described as an interaction of inertial waves, so it should be possible in principle to understand the main features of rotating turbulence (anisotropy growth, structure formation, etc.) from the interplay of turbulence and inertial waves. Inertial waves are anisotropic, dispersive waves that propagate because of the restoring nature of the Coriolis force. We first briefly describe here their main properties, and we refer the reader to classical textbooks for more details $[6,16]$.

The Navier-Stokes equations for a fluid placed in a reference frame rotating at a rate $\Omega$ are written as

$$
\left(\frac{\partial}{\partial t}-\nu \nabla^{2}\right) \mathbf{u}(\mathbf{x}, t)+\frac{\nabla p}{\rho}+(2 \Omega \mathbf{n}+\boldsymbol{\omega}) \times \mathbf{u}=0
$$

where $\omega=\nabla \times \mathbf{u}$ is the relative vorticity (in the rotating frame), $p$ the total pressure modified by the centrifugal force, $\rho$ the mass density, and $\nu$ the kinematic viscosity. The axis of rotation is borne by the unit vector $\mathbf{n}$. Inertial waves are plane-wave solutions of the form $e^{\mathrm{i}(\mathbf{k} \cdot \mathbf{x}-\sigma \mathrm{t})}$ of the inviscid linearized Navier-Stokes

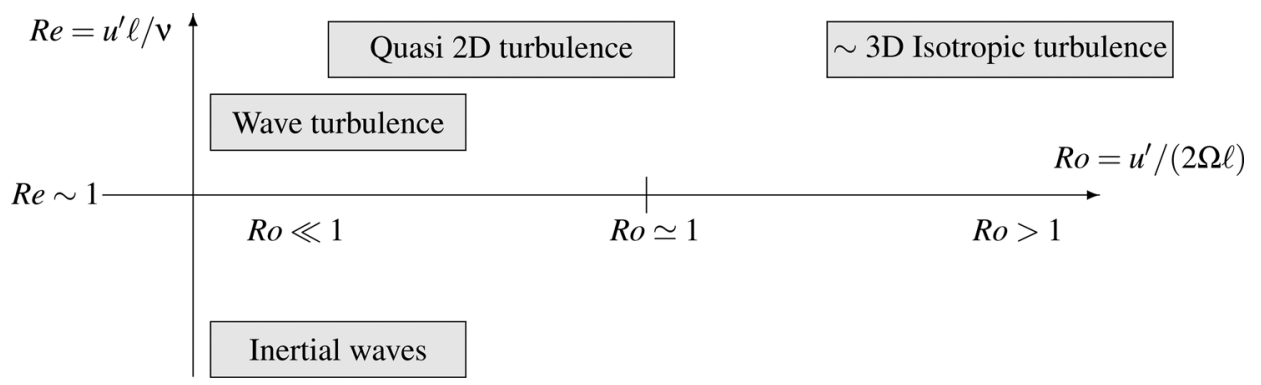

Fig. 1 Schematic of the different regimes of rotating turbulence in the Rossby-Reynolds parametric plane

030802-2 / Vol. 67, MAY 2015

Transactions of the ASME 
Eq. (1). The frequency $\sigma$ of a Fourier mode $\mathbf{k}$ is given by the dispersion relation

$$
\sigma=2 \Omega \frac{\mathbf{n} \cdot \mathbf{k}}{|\mathbf{k}|}=2 \Omega \frac{k_{\|}}{k}=2 \Omega \cos \theta
$$

where vertical - along the axis of rotation - and horizontal wavenumbers are denoted $k_{\|}=k_{z}$ and $k_{\perp}=\left(k_{x}^{2}+k_{y}^{2}\right)^{1 / 2}$, respectively, and $\theta$ is the angle between the wavevector and the vertical axis. This anisotropic dispersion relation shows that a given frequency $\sigma$ is not associated to a wavelength, but only to a direction of propagation. The frequency is maximal for wavevectors along the axis of rotation, and zero when $k_{\|}=0$. This is a first indication that vertically invariant fluid structures, composed of modes of purely horizontal wave vectors $\mathbf{k}$ (the so-called two-dimensional manifold), are stationary in the time scale of the waves: this is the essence of the Taylor-Proudman theorem [8,9]. The group velocity is

$$
\mathbf{c}_{g}=\nabla_{\mathbf{k}} \sigma=2 \Omega \frac{\mathbf{k} \times(\mathbf{n} \times \mathbf{k})}{|\mathbf{k}|}
$$

which turns out to be normal to the phase velocity $\mathbf{c}=\sigma \mathbf{k} /|\mathbf{k}|^{2}$. Accordingly, a localized disturbance of frequency $\sigma$ radiates energy along all directions allowed by $\mathbf{c}_{g}$, which form a conical wavepacket making angle $\theta$ with the horizontal, whereas the isophase surface of the wave travels across the wavepacket (the size of which is set by the disturbance size, if viscous thickening is neglected), i.e., along the wavevector. These features are nicely visualized in the early experiments of Görtler [17] (Fig. 2), in which the oblique rays mark the intersection of the conical isophase surface with the vertical plane (see Ref. [18] for similar visualizations from an oscillating linear source). In confined domains, the waves reflect on the solid boundaries and may produce inertial modes or form attractors [19] depending on the orientation of the boundaries.

If inertial waves are of vanishing amplitude, i.e., if their Rossby number $\operatorname{Ro}=u /\left(2 \Omega \ell\right.$ ) is small (where $\left.\ell \simeq k^{-1}\right)$, they propagate without interaction. But waves of finite amplitude (i.e., finite Ro) can interact nonlinearly by exchanging energy with one another.
In the low Rossby number limit Ro $\ll 1$, resonant triadic interactions occur between three inertial waves if

$$
\mathbf{k}_{1}+\mathbf{k}_{2}+\mathbf{k}_{3}=\mathbf{0} \quad \text { and } \quad \sigma\left(\mathbf{k}_{1}\right) \pm \sigma\left(\mathbf{k}_{2}\right) \pm \sigma\left(\mathbf{k}_{3}\right)=0
$$

(see, e.g., Ref. [6]). If we consider now a large ensemble of random inertial waves, it is possible to derive under the assumption of stationarity (constant energy flux supplied by an energy injection at some prescribed scales) solutions in terms of energy spectra: this is the regime of wave turbulence, explored in several contexts in physics [20]. For the particular case of isotropic waves (e.g., surface waves), Kolmogorov-Zakharov spectra of the form $k^{-\alpha}$ may be derived, but these do not account for the anisotropic nature of inertial waves.

When the Rossby number is not vanishingly small, nearresonant interactions, satisfying a less stringent resonance condition $\sigma\left(\mathbf{k}_{1}\right) \pm \sigma\left(\mathbf{k}_{2}\right) \pm \sigma\left(\mathbf{k}_{3}\right)=O$ (Ro), must also be considered. Resonant and near-resonant interactions are therefore assumed to play a dominant role in the dynamics of rotating turbulent flows at small but finite Rossby number [21,22]. In direct numerical simulations, the cartesian grid used in Fourier spectral algorithms is not adapted to the accurate representation of the spectral subspace described by Eq. (2), whose topology is complex. The passage from the continuous description to discrete modes is addressed by Bourouiba [23], who finds that discreteness effects are significant in direct numerical simulations at the currently available resolutions only for $\operatorname{Ro}<10^{-3}$. When considering near resonant interactions, numerical simulations are also possible, and permit to evaluate explicitly the energy transfers within three-dimensional wave interactions, and with the twodimensional manifold, which corresponds to nonpropagating horizontal modes of zero frequency. This last kind of interaction between wave turbulence and two-dimensional turbulence is an important research topic (see, e.g., Refs. [24-26]).

\section{Experimental Realizations of Rotating Turbulence}

We review here some of the main experimental realizations of rotating turbulence, with various experimental setups, and the related data obtained with different experimental techniques. (a)

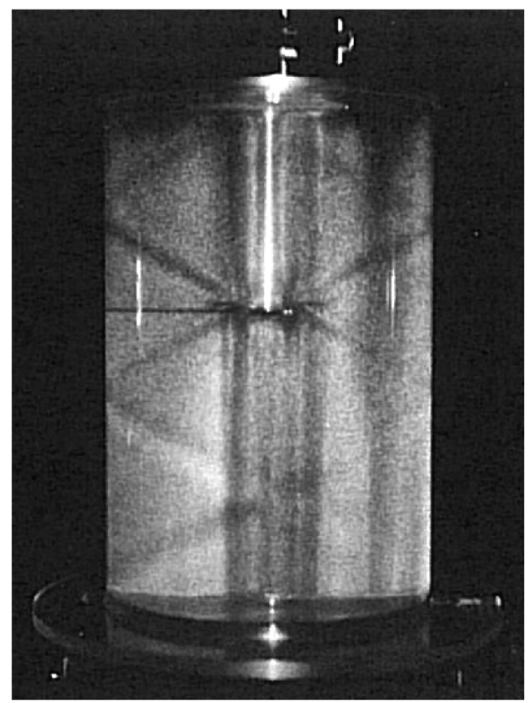

(b)

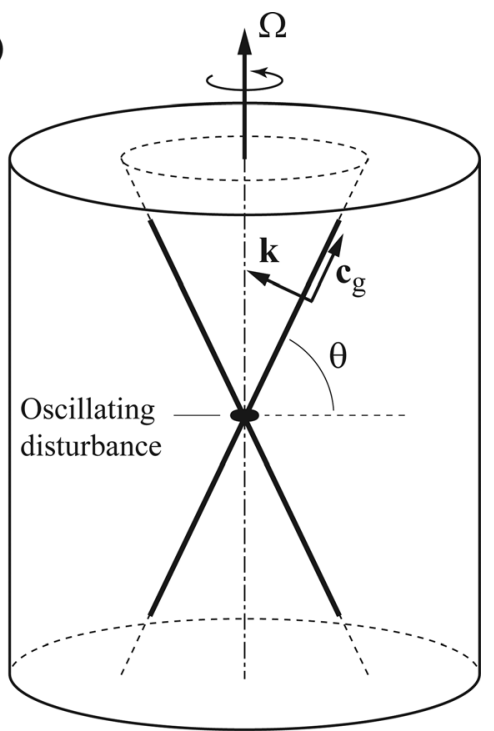

Fig. 2 Propagation of inertial waves (a) viewed in the experiment by Görtler [17]. (Reproduced with permission from Greenspan [6]. Copyright 1968 by Cambridge University.) (b) Schematic indicating the conical-shaped isophase surfaces emanating from a point source, along which velocity propagates at the group velocity $c_{g}$. 
Table 1 Recap of several rotating turbulence experiments (partially based on van Bokhoven et al. [37]). The Reynolds and Rossby numbers are based on grid parameters $\left(\operatorname{Re}_{g}, \mathbf{R o}_{g}\right)$ when relevant. Otherwise, $\operatorname{Re}_{\lambda}=u^{\prime} \lambda / v$ is the Taylor-scale-based $R e y n o l d s$ number, $\mathbf{R e}_{r}$ uses rods' thickness instead of grid mesh size, $\mathbf{R o}_{\omega}=\omega^{\prime} /(2 \Omega)$ is the micro-Rossby number based on rms vorticity, $\mathbf{R o}_{\lambda}=u^{\prime} /(2 \Omega \lambda)$ is the Rossby number based on the Taylor scale, very similar to $\mathbf{R o}_{\omega}$.

\begin{tabular}{|c|c|c|c|c|}
\hline Authors & Flow regime & Type of forcing & $\mathrm{Ro}_{g}$ & $\operatorname{Re}_{g}$ \\
\hline Ibbetson and Tritton [29] & Decay & Single grid stroke & $0.3-1.9$ & 360 \\
\hline Wigeland \& Nagib [27] & Spatial decay & Grid in wind tunnel & $6-500$ & $900-5500$ \\
\hline Hopfinger et al. [35] & Diffusive & Oscillating grid & $3-33$ & 1000 \\
\hline Dickinson and Long [36] & Diffusive & Oscillating grid & $1.4-12$ & $3800-5900$ \\
\hline Jacquin et al. [28] & Spatial decay & Grid in wind tunnel & $4-95$ & $\operatorname{Re}_{\lambda}=10-500$ \\
\hline Dalziel [30] & Decay & Single grid stroke & 0.6 & 7500 \\
\hline Baroud et al. [38] & Forced & Jets and sinks & $\mathrm{Ro}_{\omega}=0.06-1.1$ & $\operatorname{Re}_{\lambda}=360$ \\
\hline Morize et al. [31] & Decay & Single grid stroke & $2.4-120$ & $31000-62000$ \\
\hline Davidson et al. [34] & Diffusive & Single oscillation & $\mathrm{Ro}_{\omega}=1.5-3.5$ & $\mathrm{Re}_{r}=600$ \\
\hline Staplehurst et al. [32] & Decay & Single grid stroke & $\operatorname{Ro}_{\omega}=1-2.7$ & $\mathrm{Re}_{r}=83-130$ \\
\hline Van Bokhoven et al. [37] & Forced & Electromagnetic & $\mathrm{Ro}_{\lambda}=0.01-0.15$ & $\operatorname{Re}_{\lambda}=90-240$ \\
\hline Moisy et al. [33] & Decay & Single grid stroke & $\mathrm{Ro}_{g}=5-20$ & $\operatorname{Re}_{g}=42000$ \\
\hline
\end{tabular}

3.1 Experimental Setups. Several experimental efforts have been devoted to producing flows with rotation, with various practical solutions. We describe here some of them, with emphasis on the recent ones performed on rotating platforms. We restrict our list to experiments satisfying, or at least approaching, the conditions of homogeneity. They are summarized in Table 1, with typical ranges of Reynolds and Rossby numbers.

Rotating turbulence experiments can be classified in two categories, decaying and forced:

(1) Decaying rotating turbulence. In all experimental realizations of decaying rotating turbulence, turbulence is generated by a grid, which ensures a reasonable degree of homogeneity of the initial conditions. Two types of configurations have been explored: in wind-tunnel with axial mean flow $[27,28]$, and in a closed tank mounted on a rotating platform in which a grid is translated in the fluid initially at rest. Strictly speaking, the first configuration belongs to the forced turbulence category; but sufficiently far downstream the grid, and away from the lateral boundaries, the mean flow is uniform, which conveniently allows one to interpret the spatial decay in the laboratory frame as a temporal decay in the frame moving with the mean velocity. Figure 3(a) shows the wind-tunnel setup used by Jacquin et al. [28], in which rotation is produced by a rotating honeycomb mounted upstream of a grid. The first attempt to use a translated grid on a rotating platform was achieved by Ibbetson and Tritton [29], using air as working fluid. Since Dalziel [30] (see Fig. 3(b)), all experiments are now based on water-filled tanks mounted on rotating platforms [31-33,40], mainly because of measurement constraints (discussed below). Note the decaying but inhomogeneous configuration proposed by Davidson et al. [34], based on a single grid stroke in a small region of the fluid, in which the diffusion of an initially localized turbulent cloud is modified by the rotation.

(2) Forced rotating turbulence. Contrarily to decaying turbulence, which may be approximately homogeneous, forced turbulence is unavoidably inhomogeneous because of the spatial transport of energy from regions where energy is supplied. The turbulent Reynolds and Rossby numbers are now constant in time, but spatially decaying. An interesting subcase is the "diffusive" turbulence configuration, in which the energy-input device produces essentially no mean flow. This can be achieved by means of oscillating grids, first explored in the classical experiments of Hopfinger et al. [35] (see Fig. 3(c)) and Dickinson and Long [36] (see Refs. [41] and [42] for more recent realizations, using fractal grids). Other forcings have been used with similar results, like arrays of tubes acting as sinks and sources
[43-45] and electromagnetic forcing (see Fig. 3(d)) $[37,46,47]$. In these latter experiments, an array of magnets is placed below a container filled with a $\mathrm{NaCl}$ solution, with two elongated electrodes positioned near the bottom at opposite sidewalls, inducing vertical vortices of alternate sign. In all cases, the forcing device is homogeneous in a plane normal to the rotation axis, so turbulence can be considered as approximately homogeneous in horizontal planes, while decaying along the rotation axis. Recently, a new forcing has been proposed in Ref. [48], which consists of a set of vertical flaps arranged in a circle, continuously injecting turbulent jets toward the center. In this configuration, turbulence is approximately homogeneous along the vertical and statistically axisymmetric. Forced rotating turbulence can also be produced in a corotating von Karman experiments, in which two rotors placed in a cylinder propel the fluid in the same direction [49]. Turbulence lies within a large-scale coherent vortex which can be locally assimilated to a global applied rotation, although the flow is far from being homogeneous.

A common difficulty in rotating turbulence experiments is to reach simultaneously large Reynolds numbers $\operatorname{Re}=u^{\prime} \ell / \nu$ and moderate to small Rossby numbers Ro $=u^{\prime} / 2 \Omega \ell$, where $u^{\prime}$ is the characteristic turbulent velocity (measured in the rotating frame) and $\ell$ a suitably defined integral scale. The turbulent velocity $u^{\prime}$ must therefore satisfy the double constraint

$$
\frac{\nu}{\ell} \ll u^{\prime}<2 \Omega \ell
$$

which requires $2 \Omega \ell^{2} / \nu=\operatorname{Re} / \operatorname{Ro} \gg 1$. The combination $\operatorname{Re} / \mathrm{Ro}$, which can be expressed as $E^{-1}(\ell / L)^{2}$, where $E=\nu /\left(2 \Omega L^{2}\right)$ is the usual Ekman number based on the characteristic size $L$ of the experiment, is therefore constrained by the size and rotation rate of the experimental facility. In decaying grid turbulence experiments, Re and Ro are decreasing functions of time, but their ratio is fixed by the initial value $\operatorname{Re}_{g} / \operatorname{Ro}_{g}$ based on the grid size and velocity.

For most rotating platforms, mechanical constraints usually limit the maximum rotation rate such that the characteristic linear velocity, $\Omega L$, is of the order of $1 \mathrm{~m} \cdot \mathrm{s}^{-1}$. Considering water as the working fluid, taking $L \simeq 1 \mathrm{~m}$ and $\ell / L \simeq 0.1$ (a minimum requirement to ensure homogeneity), experiments in a rotating platform are typically such that $\operatorname{Re} / \operatorname{Ro} \simeq 10^{4}$. Choosing $\mathrm{Ro}=0.1$ for instance (the typical value below which rotation effects are significant) yields a maximum Reynolds number of $\mathrm{Re} \simeq 10^{3}$, showing that turbulence remains unavoidably moderate in this kind of experiment. Increasing Re/Ro beyond $10^{4}$, while keeping the homogeneity requirement $\ell / L \ll 1$, is in practice extremely 
(a)

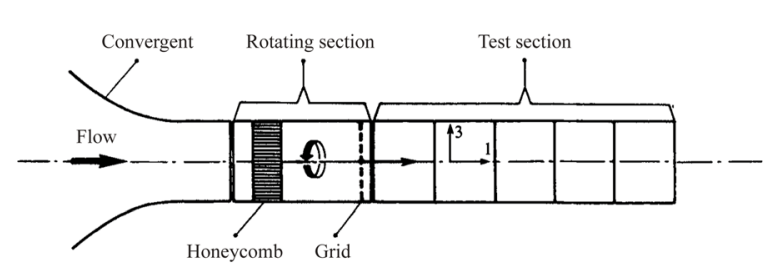

(b)

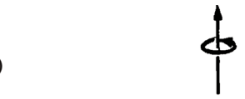

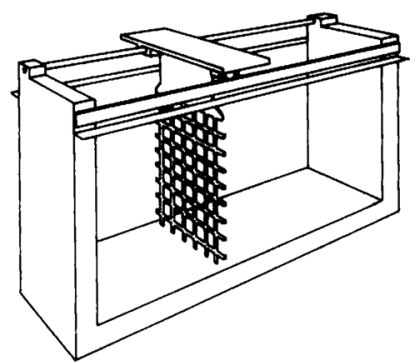

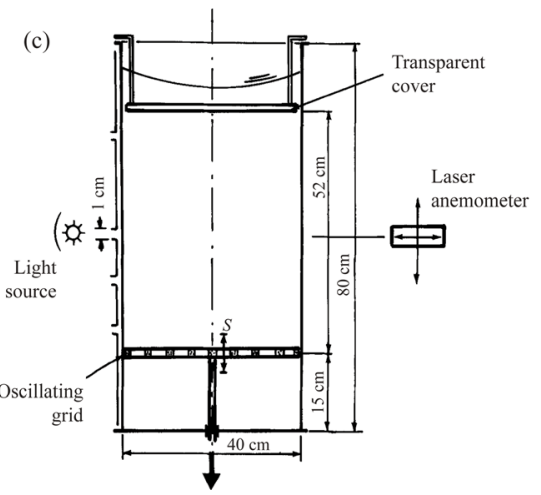

(d)

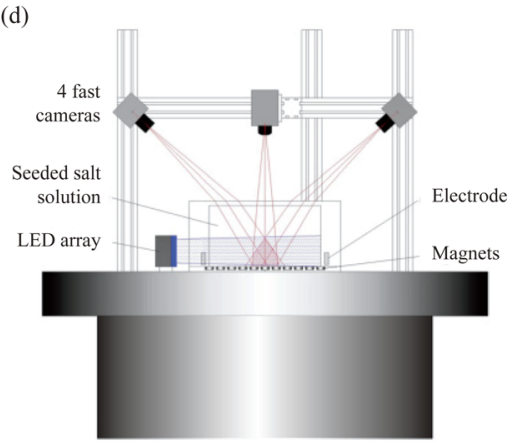

Fig. 3 Experimental configurations used for rotating turbulence: (a) rotating wind tunnel experiment developed at ONERA by Leuchter et al. (Reproduced with permission from Jacquin et al. [28]. Copyright 1990 by Cambridge University); (b) towed grid in a tank placed on a rotating platform. (Reproduced with permission from Dalziel [30]. Copyright 1992 by Springer). (c) Diffusive turbulence forced in a tank by an oscillating grid. (Reproduced with permission from Hopfinger et al. [35]. Copyright 1982 by Cambridge University). (d) Electromagnetic forcing of turbulence. Reproduced with permission from Del Castello, and Clercx [39]. Copyright 2011 by American Physical Society).

demanding. In terms of Taylor microscale Reynolds number, this corresponds to $\operatorname{Re}_{\lambda} \simeq 100$, which is often considered as a lower limit to observe inertial range scalings.

The largest rotating platform in the world is the Coriolis platform in Grenoble (France), with a diameter of $13 \mathrm{~m}$ and a maximum rotation rate of $0.05 \mathrm{~Hz}$. The maximum linear velocity is indeed of order of $1 \mathrm{~m} \cdot \mathrm{s}^{-1}$, so in spite of its considerable size the constraints on $\mathrm{Re}$ and Ro discussed above remain relevant even for this facility. Its size has nonetheless proved to be very useful for flow configurations where large aspect ratios and/or stratification are needed (e.g., Refs. [4] and [33]). In recent years, a series of rotating platforms of smaller diameters have been set up in different laboratories, with comparable values of Re/Ro, in Austin (Texas) [38], Orsay (France) [31,50], Cambridge (United Kingdom) [34], Turin (Italy) [51], Eindhoven (Netherlands) [37], Zurich (Switzerland) [41], Tel Aviv (Israel) [44], etc. (see Table 1).

An important concern for turbulence generated in closed rotating containers, even for experiments performed with a towed grid, is that inhomogeneities are unavoidably present. These inhomogeneities can be in the form of inertial modes produced by the interaction of inertial waves with the boundaries, or from large-scale motion produced by the stirring device, so that the resulting inhomogeneity is argued to be an inherent feature of any real system [30,52]. From a Reynolds decomposition performed over a large set of decay realizations, Lamriben et al. [50] showed that the spatial structure of the (time-dependent) ensemble-averaged flow was indeed given by the resonant eigenmodes of the container. It is possible, however, with some additional experimental precaution, to reduce those undesirable modes to improve the flow homogeneity.
3.2 Image-Based Velocimetry. The wealth of recent available facilities with rotating platforms also reflects the development of recent measurement techniques that permit a fine characterization of rotating turbulence. In order to characterize the anisotropic structure of the flow, measurements of two-point correlations or integral scales are necessary along the rotation axis and in the orthogonal direction. Correlations along the rotation axis have been obtained from one-point measurements (hot-wire anemometry) in the early wind-tunnel experiments [27,28], by making use of the Taylor hypothesis. But this approach was clearly limited, and did not allow to investigate the geometrical features of rotating turbulence.

The development in the last two decades of quantitative imagebased diagnostics, namely, particle image velocimetry (PIV) and particle tracking velocimetry (PTV), has had considerable impact on experimental fluid mechanics in general, and paved the way for a better multiscale evaluation of the structure and dynamics of rotating turbulence. Both techniques are based on images of small neutrally buoyant tracer particles seeding the fluid. In PIV, the particles are illuminated by a thin laser sheet, and the Eulerian velocity fields are computed from correlations of two images separated by a small time interval. It allows for the measurement of two components (with a single camera) or three components (with two cameras in stereoscopic configuration) in the plane of the laser sheet, referred to as $2 \mathrm{C} 2 \mathrm{D}$ and $3 \mathrm{C} 2 \mathrm{D}$ measurements, respectively. PTV, on the other hand, allows for three-dimensional reconstruction of particle trajectories, and requires at least 2 cameras and a powerful light source-laser, light emitting diode (LED), Xenon discharge tube [53] - illuminating a volume of fluid (see Fig. 3(d)). This method provides 3C3D Lagrangian measurements, which could be remapped onto 3C3D Eulerian 
Table 2 Excerpts of a few direct numerical simulations showing the evolution of the parameters. The forcing Rossby number is $\left.\mathbf{R o}_{f}=\left(\varepsilon_{f} \boldsymbol{k}_{f}^{2}\right)^{1 / 3}\right)$, based on dissipation and peak forcing wavenumber; when hyperviscosity is used, no Reynolds number is computed [24]. ABC forcing is the Beltrami large scale helical forcing [56]. In decaying cases, the range of initial Rossby number is shown, since it decreases in time. The simulations by Yeung and Zhou are purposely studied in a transient unsteady stage to compare the effect of rotation to that of the forcing.

\begin{tabular}{|c|c|c|c|c|c|}
\hline Authors & Flow regime & Type of forcing & Max. resolution & Ro & $\operatorname{Re}$ \\
\hline Yeung and Zhou [57] & Unsteady & Stochastic at large scales & $256^{3}$ & $0.004-0.06$ & $\operatorname{Re}_{\lambda} \simeq 140$ \\
\hline Smith and Waleffe [24] & Forced & Gaussian about peak $k_{f}$ & $200^{3}, 256^{2} \times 40$ & $\mathrm{Ro}_{f}=0.12,0.3$ & Hyperviscosity \\
\hline Morinishi et al. [58] & Decay & $\mathrm{n} / \mathrm{a}$ & $128^{3}$ & 200 & 53 \\
\hline \multirow[t]{2}{*}{ Thiele and Müller [59] } & Steady & Frozen large scales & $512^{3}$ & $0.013,0.0053$ & 2300,4000 \\
\hline & Decay & $\mathrm{n} / \mathrm{a}$ & $256^{3}$ & $0.02, \infty$ & $300-1100$ \\
\hline Teitelbaum and Mininni [60] & Decay & $\mathrm{n} / \mathrm{a}$ & $512^{3}$ & $0.07,0.1$ & 450,530 \\
\hline Yoshimatsu et al. [61] & Decay & $\mathrm{n} / \mathrm{a}$ & $256^{3}$ & $0.119-0.596$ & $56.6-93.2$ \\
\hline Mininni et al. [56] & Steady & $\mathrm{ABC}$ & $3072^{3}$ & 0.07 & 27000 \\
\hline Delache et al. [62] & Decay & $\mathrm{n} / \mathrm{a}$ & $1024^{3}$ & $0.02-0.51$ & $1590-4777$ \\
\hline
\end{tabular}

grids, although with a much lower spatial resolution than that obtained with PIV.

The first attempt to perform PIV measurements for experiments set up on a rotating platform is due to Dalziel [30], a configuration which has since received considerable interest. Ten years later, the second attempt was carried out by Baroud et al. [38] in a forced rotating turbulence experiment. Since then, 2C2D-PIV, 3C2D-PIV and 3C3D-PTV are common tools used in all the above-mentioned rotating platform experiments. Note that multiplane scanning of 2C2D PIV has been performed by Praud et al. [4], yielding 2C3D measurements, in rotating stratified experiments.

The temporal resolution of PIV and PTV is usually not an issue for experiments in rotating platforms, for which the time scale is constrained by the rotation rate of the platform. On the other hand, the modest spatial resolution of these methods, which is constrained by the particle density and the number of pixels of the cameras, is a strong limitation. In most experiments, the Kolmogorov scale, which is typically of the order of $0.1 \mathrm{~mm}$ in water experiments, is hardly resolved. The resulting limited spatial sampling leads to Eulerian velocity fields defined on grids of order of $100^{2}$ vectors only, which is usually sufficient to compute velocity correlations or structure functions, but which is clearly limiting for small-scale quantities such as vorticity and dissipation. Computation of spectral quantities from PIV is also delicate because of strong finite size effects.

\section{Numerical Simulations of Rotating Turbulence}

Introducing the Coriolis force in numerical simulations can be much easier than in experiments. For instance, from an existing code that can treat periodic turbulent flows with a semiconservative scheme for nonlinear terms as in Eq. (1), one only needs to add the background rotation $2 \Omega$ to the flow vorticity, although numerical schemes can be adapted to the rotating case with respect to the isotropic one [54]. Care must, however, be taken when considering spectral computations in periodic domains, since in that case the rotation axis is required to be along one periodic direction, in order for the periodicity condition to be consistently treated. Thus, to our knowledge, very few simulations have been performed with an inclined axis, a situation which could be of interest when an additional coupling is considered, for instance with gravity in density-variable flows, or in conducting fluids with an externally applied magnetic field. This can be the case in astrophysical situations [55]. Another issue with spectral simulations and periodic domains is the propagation of inertial waves, since they propagate away from the initial source with a velocity $c_{g}=\left|\mathbf{c}_{g}\right|$ which is of the order $\Omega \ell$ for an eddy of size $\ell$. Therefore, most simulations with periodic boundary conditions may be biased by a limited extent of the spatial domain, for times larger than the eddy turnover time. In experiments, a similar issue is present, which manifests by multiple reflexions of inertial waves at the boundaries. However, while, in experiments, the axial growth of turbulent structures is quenched by the presence of top and bottom lids, in pseudospectral simulations the periodicity of the domain enhances this growth. This is due to the fact that the velocity field is described using periodic functions, and, at large scale, numerical truncation can artificially impose unphysical vertical periodicity.

Table 2 gathers parameters of a few simulations over the last 15 years, showing the chronological evolution of the parameters; there are of course many other teams doing simulations of rotating flows. Currently, the largest available simulations are run by the group of Mininni and Pouquet with about thirty billion points at a very large Reynolds number $R e \simeq 27,000$ [56]. Forcing is often used since much larger Reynolds numbers are reached in forced turbulence compared with decaying turbulence. In the work by Mininni et al. [13], for instance, the forcing is done at large scales by imposing a Beltrami $\mathrm{ABC}$ flow (a kind of three-dimensional Taylor-Green base flow), while other authors prefer to use stochastic forcing, either at large scales or at an intermediate one. It is also worth noting that most simulations are run in a cubic domain, although it is interesting to investigate different aspect ratio boxes, preferentially extended in the axial direction, to accommodate the axial growth of the structures, or flattened boxes, as in the work by Smith and Waleffe $[24,63]$.

\section{Columnar Structures and \\ Quasi-Two-Dimensionalization in Rotating Turbulence}

As mentioned in the Introduction, anisotropy in rotating homogeneous turbulence manifests itself in the form of elongated structures along the axis of rotation, of a modified energy cascade, and of spectral scalings modified with respect to isotropic turbulence.

Initially isotropic turbulence submitted to rotation is observed to organize in columnar structures, that is, structures elongated along the axis of rotation, as shown in Fig. 4 for freely decaying turbulence in experiments $[32,33,64]$ and direct numerical simulations $[61,65]$, but also in forced rotating turbulence at various resolutions [57,59]. The quantitative statistical characterization of the elongation of structures can be done using the length scales associated with directional two-point velocity correlations

$$
L_{i i}^{j}=\frac{1}{\left\langle u_{i}^{2}\right\rangle} \int\left\langle u_{i}(\mathbf{x}) u_{i}\left(\mathbf{x}+r \mathbf{e}_{j}\right)\right\rangle d r
$$

where $\mathbf{e}_{j}$ is the unit vector along the $j$ th direction. In this way, for decaying turbulence from an initially isotropic state, the vertical scale $L_{11}^{3}$ increases much faster than the horizontal one $L_{11}^{1}$, and their separation occurs for Ro $\sim O(1)$ [66].

All results in the above-mentioned references show that the Rossby number Ro needs to be smaller than unity for elongated structures to begin appearing. This anisotropy growth corresponds to a progressive decrease of variations $\partial / \partial z$ along the axis of 

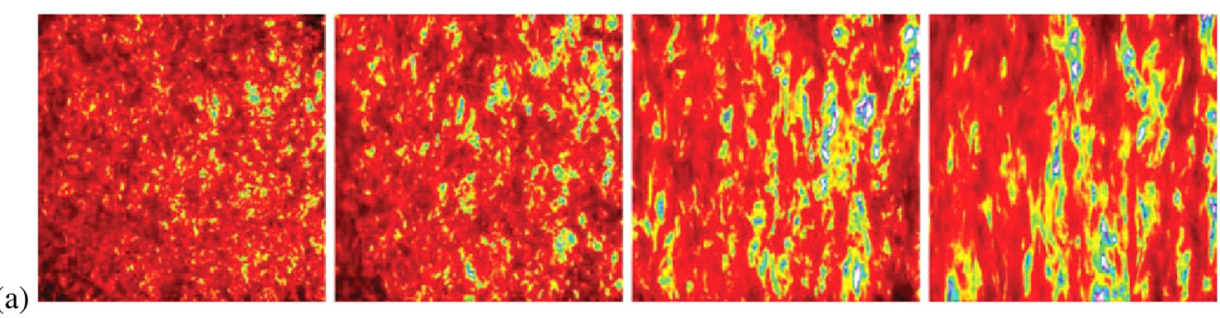

(b)
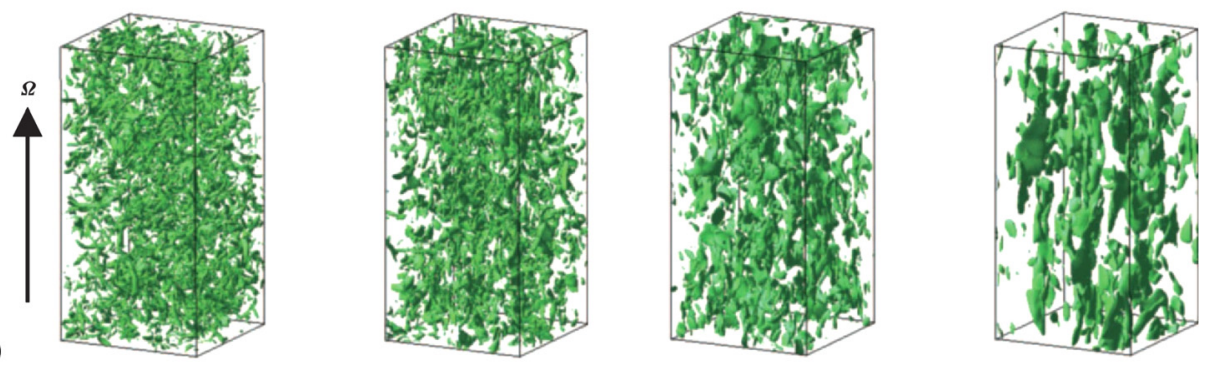

Fig. 4 Organization of turbulence in columnar structures: (a) visualization by pearlescence technique in an experiment by Staplehurst (see Table 1), as reported by Dalziel (false colors are applied to enhance the features of the flow). Turbulence decays behind a stroke of vertically towed grid, from left to right after $0.5,1,2,4$ inertial times $\Omega / 2 \pi$ after the grid left bottom of view. (Reproduced with permission from Dalziel [64]. Copyright 2011 by Cambridge University). (b) Isosurface of intense vorticity regions in a subregion of a $256^{3}$ Direct Numerical Simulation by Yoshimatsu et al., from left to right at initial time then at $\Omega t=5$ at which $R o_{\omega} \simeq 1$, and at $\Omega t=10$ and 20. (Reproduced with permission from Yoshimatsu [61]. Copyright 2011 by Cambridge University).

rotation [33]. The transition appearing at Ro $\sim O(1)$ corresponds to a progressive extension of the range of scales at which the linear effect of rotation dominates over nonlinear diffusion. This begins in the large scales and extends progressively to smaller scales, in the case of decaying turbulence. When all scales begin to be affected by rotation, that is when the microscale Rossby number $\operatorname{Ro}_{\omega}=\omega^{\prime} /(2 \Omega) \sim O(1)$, where $\omega^{\prime}$ is the $r m s$ vorticity, the complete transition of the turbulent field to a quasi-twodimensional state begins $[66,67]$. However, when considering the limit $\operatorname{Re} \rightarrow \infty$ and $\mathrm{Ro} \rightarrow 0$, the exactly two-dimensional state is not achieved at finite times in infinite or periodic domains, since the energy keeps accumulating in a neighborhood $k_{\|} \simeq 0$, but with a zero transfer rate at $k_{\|}=0$. [10].

The two-dimensional manifold $k_{\|}=0$ is, therefore, assumed to play a particular role in the dynamics of rotating turbulence. Exactly two-dimensional flows are flows in which energy is only contained in the manifold characterized by a Dirac function $\delta\left(k_{\|}\right)$ in wavenumber space, thus behaving with a two-dimensional dynamics [68]. In rotating turbulence, the two-dimensional manifold is included in three-dimensional space, so that both twodimensional and three-dimensional modes are coupled, although the precise nature of this coupling is still investigated at the level of inertial wave-vortex interactions. The corresponding energy transfers were investigated by forced numerical simulations by Smith and Waleffe [24] and in decaying turbulence simulations by Bourouiba and Bartello [25]. In the former simulations, energy is transferred to the large scales from the smaller forced ones, thus feeding the two-dimensional dynamics. In the decaying simulations, a maximum of energy transfer is observed, when varying the Rossby number, in the intermediate Ro regime for $\mathrm{Ro} \simeq 0.2$, and is the result of interactions between inertial waves and vortical structures. In statistical models of inertial wave-turbulence, as a limit of rotating turbulence when Ro $\rightarrow 0$, an explicit coupling with two-dimensional modes would have to be explicitly introduced to reproduce the transfer of energy toward the slowmanifold, and thus reproduce the wave-vortex interactions [69].

Although the anisotropization of rotating turbulence appears rather clearly from the observation of structures elongated along the rotation axis, its detailed statistical characterization requires to consider the dimensionality and the componentality of the flow, quantified by dedicated statistics. In short, in the case of rotating flows, dimensionality reflects the accumulation of energy into quasi-two-dimensional motion, and componentality is needed to sort the kind of structures that receive this energy: jetlike or vortexlike. Similar refined statistics were introduced for flows with general distortions other than rotation [70]. Componentality appears upon computing the Reynolds stress tensor anisotropy

$$
b_{i j}=\frac{\delta_{i j}}{3}-\frac{\left\langle u_{i} u_{j}\right\rangle}{\left\langle u_{k} u_{k}\right\rangle}
$$

which is important for assessing the preferential directions in which the energy of the flow accumulates. This quantity is easily accessible in experiments. $b_{i i}=0$ in incompressible flows, and, for isotropic turbulence, $b_{i j}=0$. For axisymmetric turbulence, $b_{11}=b_{22}$, so that $b_{33}=-2 b_{11} . b_{33}$ is also a relevant anisotropy component, since it not only reflects the axial fluid motion but also contains an important information related to the way energy accumulates in the neighborhood of the two-dimensional manifold.

The important difference between dimensionality and componentality may be understood from the helical wave decomposition of the velocity field. Helical modes are the two eigenmodes $\mathbf{N}(\mathbf{k})$ of the curl operator, given a wavevector $\mathbf{k}$, and were introduced first in the context of isotropic turbulence by Craya [71], then in axisymmetric and stratified flows by Herring [72], and later used in order to analyze the effect of the Coriolis term on turbulence $[66,73]$. For the sake of brevity, we shall refer to, e.g., Sagaut and Cambon [5] for details, but in short, based on the helical mode decomposition, one is able to define three contributions to the two-point velocity correlation spectrum: (1) a part that contains the energetic dependence on the wavevector orientation, $e(\mathbf{k}) ;(2)$ a polarization spectrum $Z(\mathbf{k})$ that permits to assess the preeminence of one helical mode component of velocity over the other, so that when $k_{\|}=0$, one is able to differentiate the axial contribution-vertical energy-from the horizontal contribution to horizontal kinetic energy spectrum; (3) the helicity spectrum $h(\mathbf{k})$. 

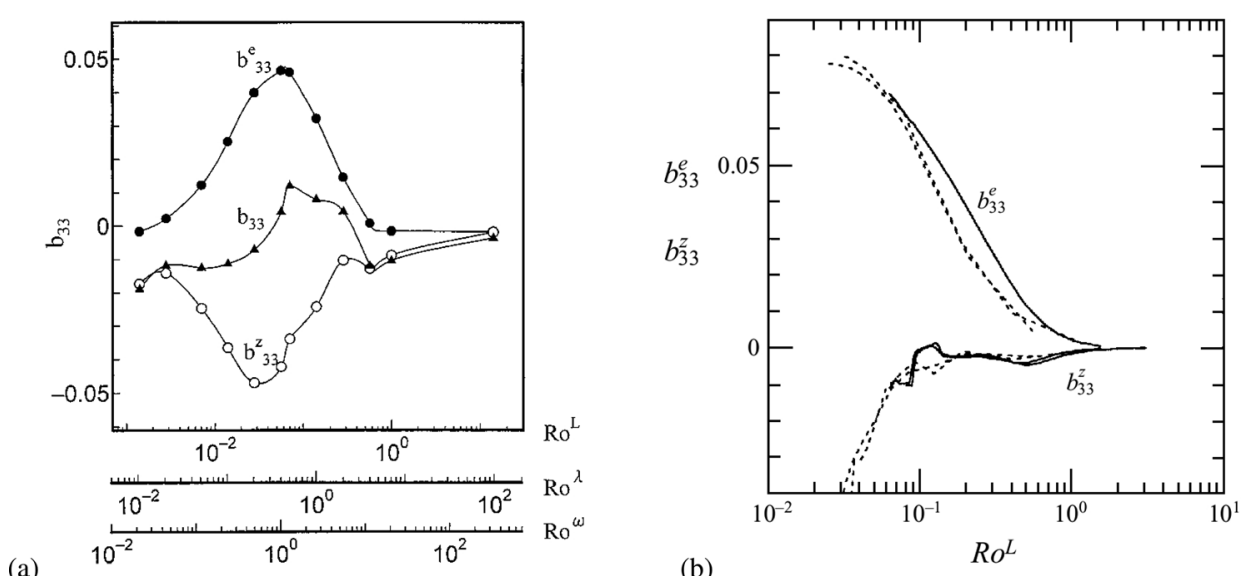

Fig. 5 Evolution of the contributions $b_{33}^{e}$ and $b_{33}^{z}$ to the Reynolds stress anisotropic component $b_{33}$ from (a) freely decaying simulations by Morinishi et al. (Reproduced with permission from Morinishi et al. [58]. Copyright 2001 by American Physical Society. (b) Simulations and EDQNM (Eddy Damped Quasi-Normal Markovian) model (dashed lines) presented in Cambon et al. (Reproduced with permission from Cambon et al. [66]. Copyright 1997 by Cambridge University). Time evolves from right to left as Rossby numbers decrease during the evolution.

The latter is assumed here to be negligible, although this may not be true in some simulations with specific helical forcing, as the $\mathrm{ABC}$ one [56]. The value of the $e, Z$ decomposition lies in the associated dynamical equations

$$
\begin{gathered}
\left(\partial_{t}+2 \nu k^{2}\right) e(\mathbf{k})=T^{e}(\mathbf{k}) \\
\left(\partial_{t}+2 \nu k^{2}-\mathrm{i} 4 \Omega \cos \theta\right) Z(\mathbf{k})=T^{z}(\mathbf{k})
\end{gathered}
$$

where $i^{2}=-1$, and in which the right-hand-side terms are nonlinear transfer terms. Equation (5) shows that the evolution of $Z(\mathbf{k})$ for horizontal modes, such that $\theta=\pi / 2$, is only driven by nonlinear terms, and no direct linear effect of rotation applies. Therefore, if all the energy of the flow were to be accumulated in the two-dimensional manifold, rotation would have no effect on its dynamics. This is consistent with the common knowledge that two-dimensional flows are not affected by rotation.

Turning back to the anisotropy tensor (3), it is possible to decompose $b_{33}$ as $b_{33}^{e}+b_{33}^{Z}$, where each contribution originates from $e(\mathbf{k})$ and $Z(\mathbf{k})$. In this decomposition, the anisotropy component $b_{33}$ contains a part which is only driven by nonlinear dynamics (Eq. (4)) and a part that may still be affected by linear dynamics (Eq. (5)). The introduction of this decomposition is justified when one computes the evolution of the two contributions in direct numerical simulations as shown on Fig. 5.

Figure 5(a) shows that, from initial isotropy with $b_{33}=0$ at large Rossby number, when Ro $\simeq O(1)$ the flow initially evolves to a more anisotropic state due to the increase of $b_{33}^{e}$; this is confirmed by Figure 5(b). Then, the plots show a decrease of anisotropy due to the drop of $b_{33}^{z}$, which can approximately be related to the crossing $\operatorname{Ro}_{\omega} \simeq O(1)$, especially on Fig. 5 (value confirmed by other statistics in Ref. [66]). Therefore, it seems that the anisotropy of the flow evolves over two timescales, one over which energy accumulates in an anisotropic way, after which the finegrained anisotropic structures of turbulence begin to appear, each effect loosely associated with linear and nonlinear processes, respectively, as hinted by Eqs (4) and (5). Unfortunately, it is impractical up to now to obtain experimental information about $b_{33}^{e}$ and $b_{33}^{z}$, since there is no means of obtaining the separate polarization spectral components in the two-point velocity correlation tensor from experimental data.

\section{Cyclone-Anticyclone Asymmetry}

During the process of anisotropy growth and columnar structure formation, the distribution of elongated vortices becomes asymmetric, such that cyclonic vorticity is favored. Cycloneanticyclone asymmetry is a generic feature of rotating flows, which originates from the modification of stretching and tilting of the vorticity by the Coriolis force, suggesting a more pronounced asymmetry at $\mathrm{Ro} \simeq O(1)$. In homogeneous rotating turbulence the cyclonic-anticyclonic asymmetry is reflected in the asymmetry of the pdf (probability density function) of $\omega_{z}$, as shown in experimental data on Fig. 6 [31]. The statistical characterization of this asymmetry may be done through the observation of a nonzero value of the third-order moment of axial vorticity $\left\langle\omega_{z}^{3}\right\rangle$ (or its skewness $S_{\omega}=\left\langle\omega_{z}^{3}\right\rangle /\left\langle\omega_{z}^{2}\right\rangle^{3 / 2}$ ) [32,74,75].

The asymmetry is expected to strongly depend on the details of the forcing and boundary conditions. A generic behavior may, however, be expected in the decaying case, starting from initially isotropic turbulence (such that $\operatorname{Re} \gg 1$ and Ro $>1$ at initial time). Experimental measurements [31-33] and numerical simulations [61,75] indeed show similar behaviors (Fig. 7), with a rather reproducible growth of the vorticity skewness, close to a power law growth. However, the nonlinear behavior that follows this initial stage can vary between the different flow cases, with a saturation or a decrease at large time. This second stage apparently depends on the way the flow is initially generated, and may be sensitive to the boundary conditions, Rossby and Reynolds numbers.

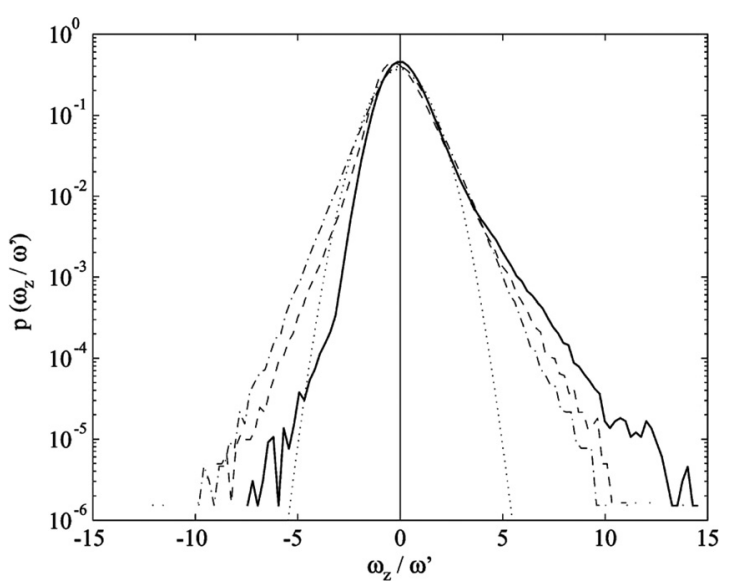

Fig. 6 PDF of axial vorticity by Morize et al. (Reproduced with permission from Morize et al. [31]. Copyright 2005 by American Physical Society). 


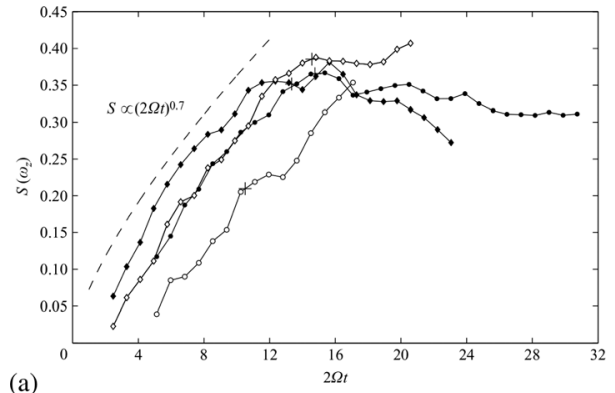

(a)

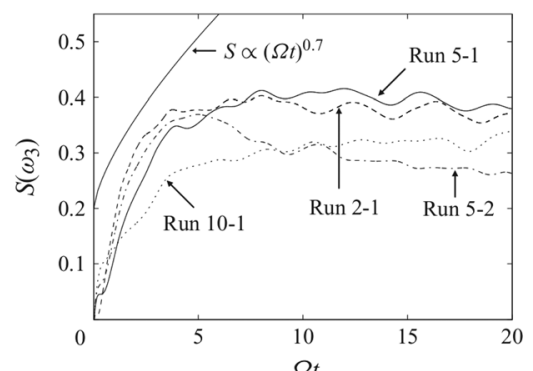

(c)

$\Omega$
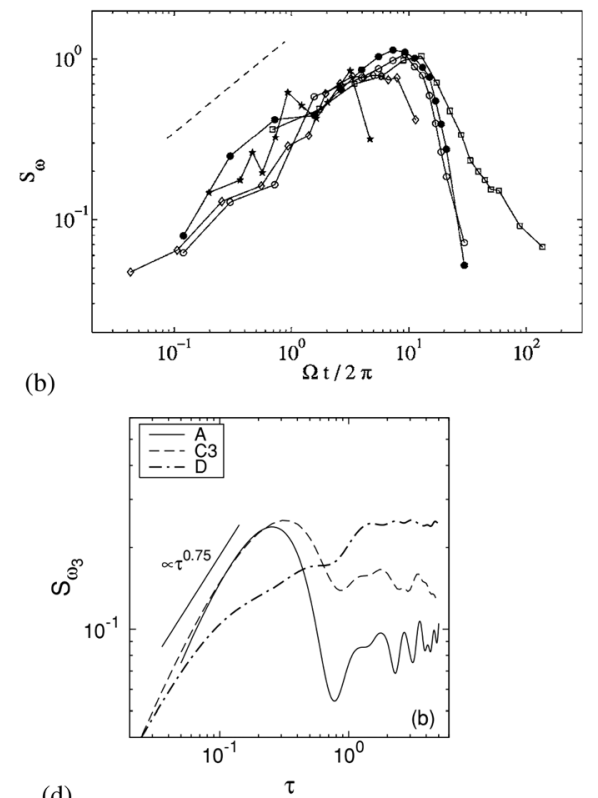

(d)

Fig. 7 Growth of axial vorticity skewness $\left\langle\omega_{z}^{3}\right\rangle /\left\langle\omega_{z}^{2}\right\rangle^{3 / 2}$ in experiments (top figures) and numerical simulations (bottom figures): (a) Experimental data by Staplehurst et al. filled and open symbols, respectively, at initial $\mathrm{Ro} \simeq 0.37$ and 0.41 , two different rotation rates used. (Reproduced with permission from Staplehurst et al. [32]. Copyright 2008 by Cambridge University.) (b) Experimental data by Morize et al. at $\mathrm{Ro}_{g}$ from 2.4 to 120. (Reproduced with permission from Morize et al. [31]. Copyright 2005 by American Physical Society). (c) DNS (direct numerical simulation) data by Yoshimatsu et al. with Ro from $4.8 \times 10^{-2}$ to 0.24 . (Reproduced with permission from Yoshimatsu et al. [61]. Copyright 2011 by Cambridge University). (d) DNS data by van Bokhoven et al. at $\mathrm{Ro}_{\lambda}$ from 0.073 to 0.37 . (Reproduced with permission from van Bokhoven et al. [75]. Copyright 2008 by Taylor \& Francis).

One explanation for the asymmetry growth is provided by the analytical derivation of Gence and Frick [76], which expresses the growth of $\left\langle\omega_{z}^{3}\right\rangle$ at short time in a suddenly rotated isotropic turbulence

$$
\frac{d\left\langle\omega_{z}^{3}\right\rangle}{d t}\left(t=0^{+}\right)=\frac{4}{5} \Omega\left\langle\omega_{i} \omega_{j} s_{i j}\right\rangle(t=0)
$$

where $s_{i j}$ is the rate of strain tensor. This predicts a linear growth of $\left\langle\omega_{z}^{3}\right\rangle$ at short time, in qualitative agreement with the observations, but the long-time behavior cannot be inferred from this equation when $s_{i j}$ becomes in turn modified by rotation. It is important to note that this process of emergence of dominant cyclonic motion from an initially isotropic state is different from the selection of cyclonic structures among pre-existing cyclonic and anti-cyclonic vortices [77]. The latter mechanism involves the stability of coherent structures, from various possible mechanisms, such centrifugal or elliptical instability.

An important open question is the scale dependence of the asymmetry: at what scales are the vortical structures that contribute to the asymmetry? Does the asymmetry first appear at small scales, or is it dominated by a few large-scale cyclones? This could be probed from filtered vorticity field [33] or two-point third-order velocity correlation $[48,63]$. The answer probably depends on whether the scales are larger or smaller than the Zeman scale (the scale at which the turbulent time is of order of the global rotation, see below), although no systematic study has been performed so far.

\section{Spectral Scalings and Scale-by-Scale Anisotropy}

From the observation of the anisotropic structure of rotating turbulence, one expects that energy spectra $E(k)$ exhibit scalings which differ from the Kolmogorov scaling $k^{-5 / 3}$ of isotropic turbulence. Moreover, one may consider not only spherical spectra that depend on the wavenumber $k$ but also spectra that exhibit a directional dependence, in the form $e\left(k_{\perp}, k_{\|}\right)$where $k_{\perp}$ is the wavenumber component orthogonal to $\Omega$, or in a similar guise $e(k$, $\theta$ ), separating scale $k$ dependence from direction dependence. In the latter case, one considers the important horizontal spectrum at $\theta=\pi / 2$, which is related to the quasi-two-dimensional dynamics, which can be obtained in the former case by setting $k_{\|}=0$.

Theoretical, experimental, and numerical results about the scaling of the kinetic energy spectrum are available. In a phenomenological study, Mahalov and Zhou [78,79] examine the different characteristic timescales appearing in the long-term behavior of rapidly rotating turbulence. They propose the following scaling (the Zhou spectrum): $E(k)=C_{\Omega}(\Omega \varepsilon)^{1 / 2} k^{-2}$ considering only the rotation timescale, for small $k$ such that $\mathrm{Ro}<1$, which is corrected in $E(k)=C_{2}\left(k_{\Omega}\right) \varepsilon^{2 / 3} k^{-5 / 3}$ when both rotation and eddy-turnover timescales are combined, for larger $k$ at Ro $>1$ ( $\varepsilon$ is the kinetic energy dissipation). In the latter estimate, the prefactor is expressed in terms of the Zeman wavenumber $k_{\Omega}=\left(\Omega^{3} / \varepsilon\right)^{1 / 2}$ that appears to be an important scale as discussed further below. When considering turbulence forced at an intermediate wavenumber $k_{f}$, as in simulations by Smith and Waleffe [24], different scalings are found above and below the forcing wavenumber: for $k<k_{f}$, $E(k) \sim k^{-3}$ as in two-dimensional turbulence, which could be a trace of an inverse cascade; for $k>k_{f}, k^{-5 / 3}$ is recovered as befits a forward Kolmogorov-like cascade down to the smallest scales. In the work by Mininni and Pouquet [13] in which turbulence is forced by a helical $\mathrm{ABC}$ flow at large scales, the energy spectrum scaling is $k^{-5 / 3}$, but another cascade intervenes for the helicity spectrum that scales as $k^{-2}$.

The fact that the small scales of rotating turbulence are strongly anisotropic can be interpreted with the help of the Zeman wavenumber $k_{\Omega}=\left(\Omega^{3} / \varepsilon\right)^{1 / 2}$, or the related Zeman length scale $2 \pi / k_{\Omega}$. Although defined a long time ago, this scale and its importance has been supported by recent investigations into the anisotropy at the various scales of rotating turbulence. These investigations 

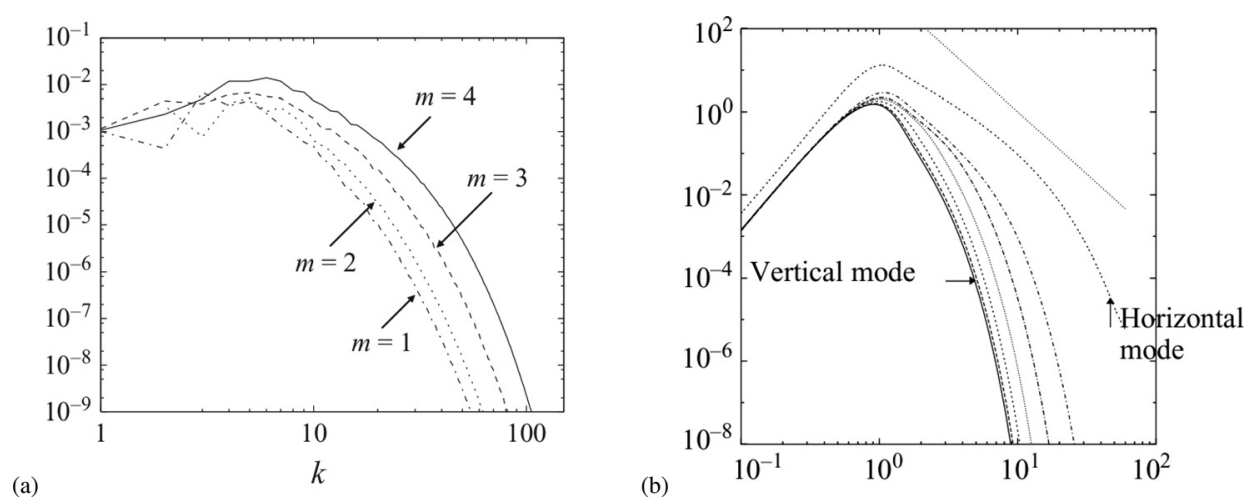

Fig. 8 Directional spectra $E(k, \theta)$ from (a) direction numerical simulations by Yoshimatsu et al. (Reproduced with permission from Yoshimatsu et al. [61]. Copyright 2011 by Cambridge University). (b) From the two-point statistical AQNM (asymptotic quasi-normal Markovian) model by Bellet et al. (Reproduced with permission from Bellet et al. [69]. Copyright 2006 by Cambridge University). The lower spectra are for the vertical direction $\theta \simeq \pi / 2$, the upper ones for the horizontal spectra at $\theta \simeq 0$. In the simulations, four angular sectors of $\theta$ are used to compute the statistical averages, whereas in the AQNM model, spectra correspond to discrete orientations.

seem to demonstrate, both in experimental results [34] and in numerical simulations $[56,62]$, that each turbulent scale can be compared to the Zeman scale to determine whether anisotropy is present or not, and that maximum anisotropy is found at scales of order of the Zeman scale. For instance, in the simulations presented in Fig. 8(a), but also in the simulations by Delache et al. [62], if $k_{\Omega}>k$ for all wavenumbers $k$ in the inertial range, rotation affects all scales and renders them anisotropic. If $k_{\Omega}$ is in an intermediate range, anisotropy affects the large scales only while isotropy is recovered for small scales. Therefore, it is necessary that $\Omega$ be large enough with respect to the dissipation $\epsilon$ for anisotropy to begin to appear, or, conversely, that the dissipation become small enough - for instance in decaying turbulence - at fixed rotation rate.

The next refined description accounts for the directional dependence of the spectra in the $\left(k_{\perp}, k_{\|}\right)$spectral space, since rotating turbulence is statistically axisymmetric. The theoretical prediction by Galtier [80] in a wave turbulence framework, agreeing with rapidly rotating turbulence, is based on the helical modes decomposition, and provides the anisotropic energy spectrum scaling $E\left(k_{\perp}, k_{\|}\right) \sim k_{\perp}^{-5 / 2} k_{\|}^{-1 / 2}$ - the $k_{\perp}$ power may vary if one takes into account a low-wavenumber spectral cut-off in the $k_{\perp}$ direction [81] - and $H\left(k_{\perp}, k_{\|}\right) \sim k_{\perp}^{-3 / 2} k_{\|}^{-1 / 2}$ for the helicity spectrum. In the asymptotic low Rossby number limit, the theoretical scalings of helicity and energy spectra are shown to be linked [82]. The theory also confirms that, to lowest order, the twodimensional modes are not dynamically coupled to the threedimensional wave-turbulence. The simulations by Mininni et al. [56] seem to confirm the $k^{-5 / 2}$ scaling of the spectrum, but only for the $k_{\|}=0$ contribution, and for scales larger than the Zeman scale. For smaller scales, the $k^{-5 / 3}$ scaling is again recovered. In nonhelically large-scale forced simulations by Thiele and Müller [59], the horizontal spectral scaling for energy is found to be $E\left(k_{\perp}, k_{\|}=0\right) \sim k_{\perp}^{-2}$, but the authors show that the scaling of the full spectrum is $E\left(k_{\perp}\right) \sim k_{\perp}^{-3}$ close to the two-dimensional manifold, and is the result of integration over $k_{\|}$, hence underlining the important difference between one-dimensional spectra and integrated spectra.

An example of directional spectral dependence with respect to $k, \theta$, which contains the same information as the $k_{\perp}, k_{\|}$dependence, is shown in Fig. 8. It shows that the largest anisotropy, in terms of horizontal-to-vertical energy difference at a given $k$, appears in the smallest scales, both in simulations (Fig. 8(a) [61]) and in a two-point statistical model (Fig. 8(b) [69]) for weak inertial wave turbulence. In the latter, the horizontal mode is not represented, so that the vertical separation of the most energetic spectrum appearing in Fig. 8(b) already hints at a decoupling of the neighborhood of the slow manifold.

\section{Balance Statistical Equations in Physical and Spectral Formalisms and Related Dynamics}

The interpretation of the above results requires a careful examination of the dynamical equations. We thus consider the balance equations for two-point statistics, obtained from Eq. (1). It permits to examine the dynamics of the flow in terms of scales, and to obtain a diagnostic of the scale-by-scale exchanges. Considering the trace of the two-point velocity correlation tensor $R=R_{i i}(\mathbf{r})$ $=\left\langle u_{i}(\mathbf{x}) u_{i}(\mathbf{x}+\mathbf{r})\right\rangle$, one derives the Karman-Howarth-Monin equation

$$
\frac{1}{2} \partial_{t} R=\frac{1}{4} \nabla \cdot \mathbf{F}+\nu \nabla^{2} R
$$

in which the flux term $\mathbf{F}=\langle\delta \mathbf{u}(\delta \mathbf{u} \cdot \delta \mathbf{u})\rangle$ is a third-order quantity based on the velocity increments $\delta \mathbf{u}=\mathbf{u}(\mathbf{x}+\mathbf{r})-\mathbf{u}(\mathbf{x})$ (see, e.g., Ref. [83]). In isotropic turbulence $\mathbf{F}$ is aligned with the separation vector $\mathbf{r}$ and, for scales $|r|$ in the inertial range, Eq. (6) integrates to the famous 4/5th law, $F_{r}=-(4 / 5) \varepsilon r$, with $\varepsilon=-d_{t} R(r=0) / 2$ the energy dissipation rate. The central question here is to model this anisotropic flux $\mathbf{F}$ in the presence of rotation.

No explicit effect of rotation appears in Eq. (6), since the Coriolis force, being orthogonal to $\mathbf{u}$ by nature, does no work, but rotation appears in the dynamical equation for $\mathbf{F}$. It therefore required phenomenological scaling arguments to Galtier [84] to be able to propose a functional dependence of the flux term with respect to the direction of the separation vector, in rotating turbulence.

The analogous of the Karman-Howarth-Monin equation in spectral space is the Lin equation, which has a similar structure, wherein the third-order terms in the right-hand-side are exactly the nonlinear energy transfer for a given wavenumber $k$ of energy $E(k)$

$$
\partial_{t} E(k)=T(k)-2 \nu k^{2} E(k)
$$

Note that the Lin equation is an integrated version of the energy density equation similar to Eq. (4), the integration over the angles being used to remove the explicit $\theta$ dependence. Note also that the other spectral equation (5) for the polarization spectrum is not reflected in Eq. (6) in terms of physical contents. 


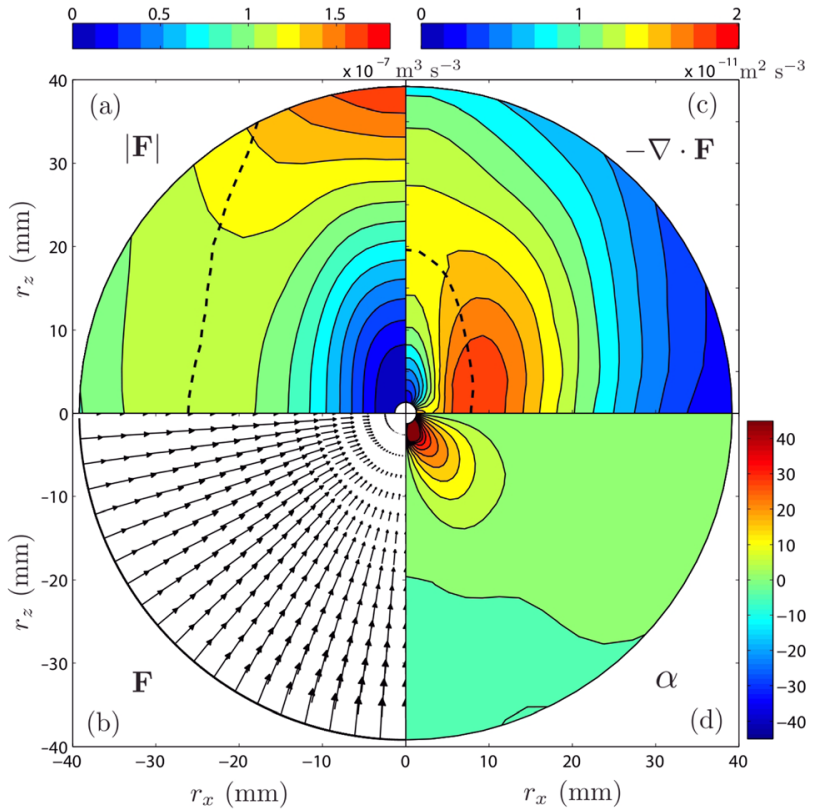

Fig. 9 Nonlinear transfer $-\nabla \cdot F$ measured experimentally by Lamriben et al. (top right sector), along with the flux itself $F$ represented as a vector field in the bottom left sector, and its angle with respect to the radial direction in the bottom right sector. Dashed lines represent crests of maxima of $|F|$ and of $-\nabla \cdot F$ in the top left and top right quadrants, respectively. (Reproduced with permission from Lamriben et al. [40]. Copyright 2011 by American Physical Society).

As mentioned in Sec. 3, the computation of the different terms in Eq. (6) has been rendered possible from experimental results when PIV techniques began to be developed. Interestingly, from direct numerical simulations, Eq. (7) only was used, since, from spectral simulation methods, one has a rather easy access to the energy transfer term $T(k)$. But this term bears the same information as $(1 / 4) \nabla \cdot \mathbf{F}$ in Eq. (6).

Obtaining experimental evaluations of the different terms in Eq. (6) requires a significant experimental effort, since third-order moments are prone to statistical noise. In a first dedicated setting using only two-dimensional measurements that permit to obtain accurate statistics over a hundred realizations, Lamriben et al. [40] were able to provide the anisotropic distribution of the different terms appearing in the Karman-Howarth-Monin equation in planes $\left(r_{x}, r_{z}\right)$. Figure 9 exhibits in the top-right quadrant the transfer term appearing in the Karman-Howarth-Monin equation, that formally corresponds to the energy transfer $T(\mathbf{k})$ of the Linequation upon Fourier transforming to spectral space. The figure demonstrates the depletion of $-\nabla \cdot \mathbf{F}$ along the axis induced by the nonlinear term, whereas an excess of $-\nabla \cdot \mathbf{F}$ is produced in the horizontal modes, in the inertial subrange. An interesting original result is that the flux vector field $\mathbf{F}\left(r_{x}, r_{z}\right)$ is only marginally offradial, so that the anisotropy of its divergence is actually mostly due to the angular dependence of its magnitude. Obtaining the equivalent of $-\nabla \cdot \mathbf{F}$ in spectral space would be possible, and may bring additional understanding of the basic anisotropization phenomenon exhibited by the directional kinetic energy transfer spectrum.

Nonetheless, the spectral viewpoint by itself can provide additional physical insight into the anisotropic phenomena. Since neither Eq. (6) nor Eq. (7) exhibit any obvious direct influence of rotation, this means that, in absence of additional inhomogeneity effects, the third-order terms contain nonlinear interactions of inertial waves, and is the only way rotation can butt in on the dynamics. These third-order terms correspond to triadic interactions in the energy transfer terms of Eq. (7), in which the action of the linear rotating term appears formally through triple products of its associated Green's function. Thus, each triadic contribution to the energy transfer is weighted by a phase-scrambling term $\exp \left[\mathrm{i}\left(\sigma\left(\mathbf{k}_{1}\right) \pm \sigma\left(\mathbf{k}_{2}\right) \pm \sigma\left(\mathbf{k}_{3}\right)\right)\right]=\exp \left[2 \Omega \mathrm{i}\left(k_{1 \|} / k_{1} \pm k_{2 \|} / k_{2}+k_{3 \|} / k_{3}\right)\right]$ so that $\Omega$ weights differently resonant and off-resonant triads. The corresponding modified dynamics in the energy transfer terms of Eq. (7) is thus more easily identified in spectral space than in physical space in Eq. (6), in which one would have to solve anisotropic pressure through the Poisson equation to account for the role of rotation. In the spectral formulation, the pressure vanishes in a simpler way from the divergence free property $\mathbf{k} \cdot \hat{\mathbf{u}}(\mathbf{k})=0$, where $\hat{\mathbf{u}}$ is the velocity Fourier component (see for instance [5]). The corresponding modification of kinetic energy transfer is reflected in the evolution of the velocity derivative skewness, for which a phenomenological dependence with the Rossby number was found to be

$$
S=\frac{\left\langle\left(\partial_{r} u\right)^{3}\right\rangle}{\left\langle\left(\partial_{r} u\right)^{2}\right\rangle^{3 / 2}}=-\frac{0.49}{\left(1+2\left(\operatorname{Ro}_{\omega}\right)^{-2}\right)^{1 / 2}}
$$

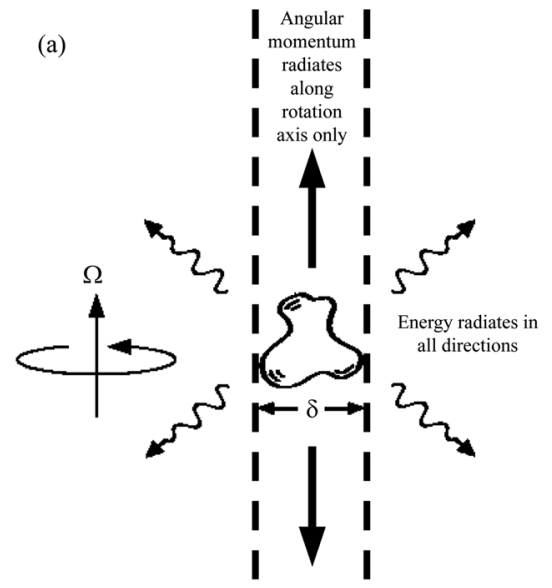

(b)

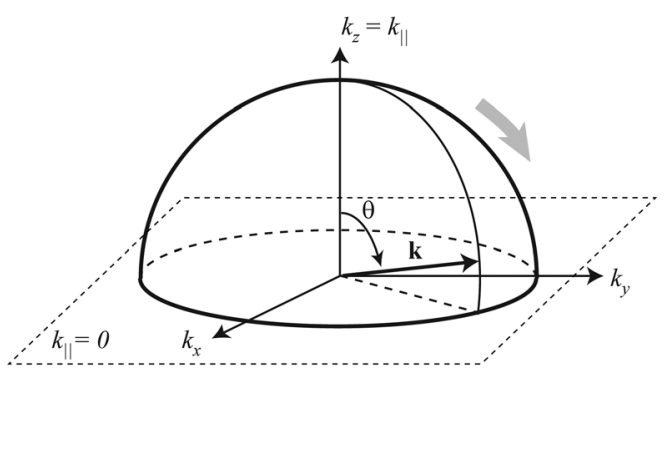

Fig. 10 Sketch of the structuration of rotating turbulence under the effects of (a) propagation of inertial waves from initial inhomogeneous turbulent structures as proposed by Staplehurst et al. (Reproduced with permission from Staplehurst et al. [32]. Copyright 2008 by Cambridge University). (b) Accumulation of energy in the vicinity of the two-dimensional manifold hence producing vertical vortices (as proposed, e.g., in Ref. [85]). 
This dependence shows that, from the classical isotropic value -0.49 at large Rossby number when rotation is weak, the skewness steadily decreases upon increasing the rotation rate. The functional dependence (8) proposed by Cambon et al. [66] from results of direct numerical simulations and of two-point statistical modeling of rotating turbulence is also well verified by experimental data [31].

It is also interesting to discuss the interpretation of the mechanism of creation of elongated structures and of anisotropy in rotating turbulence, with two points of view. On the one hand, the above mechanism explains the structuration by nonlinear phenomena transferring energy toward the two-dimensional manifold (see Fig. 10(b)); on the other hand, the interpretation proposed by Staplehurst et al. [32] based on the linear propagation of inertial waves along the rotation axis from initial turbulent patches (see Fig. 10(a)). In order to reconcile both approaches, one could propose a combination of the two mechanisms, wherein, on the one hand, turbulent blobs - originating from initial conditions or turbulence forcing mechanisms - are propagated vertically over a small timescale based on $\mathrm{Ro}^{-1}$, and, on the other hand, nonlinear interactions set in over a longer timescale based on $\mathrm{Ro}^{-2}$. Depending on the specific parameter of each flow, and considering the fact that most experiments and direct numerical simulations are still in a moderate Rossby number range Ro $=O(1)$, these two phenomena may be mingled, and can overlap for some time. This could explain the various observations and differing behaviors in experimental or numerical statistics of rotating turbulence.

\section{Conclusions}

This review contains a snapshot of selected recent results and mechanisms in the study of rotating turbulence. In terms of the structural characterization of the flow, modern metrology used in experiments permit to obtain a refined picture of not only the velocity field topology but also the axisymmetric velocity field statistics, including high-order moments. The current resolution of PIV and PTV is still not able to provide the complete small-scale dynamics, especially for a good estimate of the dissipative scales, but progress is expected in the near future. Computer power is also rapidly increasing, and the larger resolution direct numerical simulations of rotating turbulence currently provide a good separation of scales necessary to understand its complex dynamics. There is still some way though before one will be able to compute a flow for instance combining rotation and stable stratification effects in proportion of those observed in the atmospheric boundary layer, since the required scale separation and Reynolds number value are currently beyond our reach, computationally speaking. In addition to these efforts, modeling can still be improved, especially regarding the coupling between the twodimensional turbulent manifold and the three-dimensional wave field. A self-consistent theory for rotating turbulence, and, starting, for more general axisymmetric turbulence, in the guise of Kolmogorov's for isotropic turbulence, is yet to be developed.

\section{Acknowledgment}

F.M. would like to thank the Institut Universitaire de France for its support, and the Agence Nationale de la Recherche through Grant No. ANR- 2011-BS04-006-01. F.S.G. acknowledges the support of Agence Nationale de la Recherche through Grant No. ANR-08-BLAN-0076.

\section{References}

[1] Barnes, S. A., 2001, "An Assessment of the Rotation Rates of the Host Stars of Extrasolar Planets," Astrophys. J., 561(2), pp. 1095-1106.

[2] Cho, J. Y.-K., Menou, K., Hansen, B. M. S., and Seager, S., 2008 "Atmospheric Circulation of Close-in Extrasolar Giant Planets: I. Global, Barotropic, Adiabatic Simulations," Astrophys. J., 675(1), pp. 817-845.

[3] Dumitrescu, H., and Cardos, V., 2004. "Rotational Effects on the BoundaryLayer Flow in Wind Turbines," AIAA J., 42(2), pp. 408-411.
[4] Praud, O., Sommeria, J., and Fincham, A., 2006, "Decaying Grid Turbulence in a Rotating Stratified Fluid,” J. Fluid Mech., 547, p. 389.

[5] Sagaut, P., and Cambon, C., 2008, Homogeneous Turbulence Dynamics, Cambridge University, Cambridge, UK.

[6] Greenspan, H. P., 1968, The Theory of Rotating Fluids, Cambridge University, Cambridge, UK.

[7] Bennetts, D. A., and Hocking, L. M., 1973, "On Nonlinear Ekman and Stewartson Layers in a Rotating Fluid," Proc. R. Soc. London, Ser. A, 333(1595), pp. 469-489.

[8] Taylor, G. I., 1917, "Motion of Solids in Fluids When The Flow Is Not Irrotational,” Proc. R. Soc. London, Ser. A, 93(648), pp. 99-113.

[9] Proudman, J., 1916, "On the Motion of Solids in a Liquid Possessing Vorticity," Proc. R. Soc. London, Ser. A, 92(642), pp. 408-424.

[10] Babin, A., Mahalov, A., and Nicolaenko, B., 2000, "Global Regularity of 3D Rotating Navier-Stokes Equations for Resonant Domains," Appl. Math. Lett. 13(4), pp. 51-57.

[11] Zeman, O., 1994, "A Note on the Spectra and Decay of Rotating Homogeneous Turbulence," Phys. Fluids, 6(10), p. 3221.

[12] Batchelor, G. K., 2000, An Introduction to Fluid Dynamics, Cambridge University, Cambridge, UK.

[13] Mininni, P. D., and Pouquet, A., 2010, "Rotating Helical Turbulence. I. Global Evolution and Spectral Behavior," Phys. Fluids, 22(3), p. 035105.

[14] Lamriben, C., Cortet, P.-P., and Moisy, F., 2011, "Direct Measurements of Anisotropic Energy Transfers in a Rotating Turbulence Experiment," Phys. Rev. Lett., 107, p. 024503 .

[15] Davidson, P. A., 2013, Turbulence in Rotating, Stratified and Electrically Conducting Fluids, Cambridge University, New York.

[16] Lighthill, M., 1978, Waves in Fluids, Cambridge University, New York.

[17] Görtler, H., 1957, "On Forced Oscillations in Rotating Fluids," Proceedings of the Fifth Midwestern Conference on Fluid Mechanics, The University of Michigan, Ann Arbor, MI, Apr. 1-2, pp. 1-10.

[18] Cortet, P.-P., Lamriben, C., and Moisy, F., 2010, "Viscous Spreading of an Inertial Wave Beam in a Rotating Fluid,” Phys. Fluids, 22(8), p. 086603.

[19] Maas, L. R. M., 2001, "Wave Focusing and Ensuing Mean Flow due to Symmetry Breaking in Rotating Fluids," J. Fluid Mech., 437, pp. 13-28.

[20] Nazarenko, S., 2011, Wave Turbulence, Springer, Berlin.

[21] Smith, L. M., and Lee, Y., 2005, "On Near Resonances and Symmetry Breaking in Forced Rotating Flows at Moderate Rossby Number," J. Fluid Mech., 535, p. 111.

[22] Bourouiba, L., Straube, D. N., and Waite, M. L., 2012, "Non-Local Energy Transfers in Rotating Turbulence at Intermediate Rossby Number," J. Fluid Mech., 690, pp. 129-147.

[23] Bourouiba, L., 2008, "Discreteness and Resolution Effects in Rapidly Rotating Turbulence,” Phys. Rev. E, 78, p. 056309.

[24] Smith, L., and Waleffe, F., 1999, "Transfer of Energy to Two-Dimensional Large Scales in Forced, Rotating Three-Dimensional Turbulence," Phys. Fluids, 11(6), pp. 1608-1622.

[25] Bourouiba, L., and Bartello, P., 2007, "The Intermediate Rossby Number Range and Two-Dimensional-Three-Dimensional Transfers in Rotating Decaying Homogeneous Turbulence," J. Fluid Mech., 587, pp. 139-161.

[26] Scott, J., 2014, "Wave Turbulence in a Rotating Channel," J. Fluid Mech., 741, pp. 316-349.

[27] Wigeland, R. A., and Nagib, H. M., 1978, "Grid-Generated Turbulence With and Without Rotation About the Streamwise Direction," IIT Fluid and Heat Transfer Report No. R78-1, Illinois Institute of Technology, Chicago, IL.

[28] Jacquin, L., Leuchter, O., Cambon, C., and Mathieu, J., 1990, "Homogeneous Turbulence in the Presence of Rotation," J. Fluid Mech., 220, pp. 1-52.

[29] Ibbetson, A., and Tritton, D. J., 1975, "Experiments on Turbulence in a Rotating Fluid," J. Fluid Mech., 68(4), pp. 639-672.

[30] Dalziel, S., 1992, "Decay of Rotating Turbulence: Some Particle Tracking Experiments," Appl. Sci. Res., 49(3), pp. 217-244.

[31] Morize, C., Moisy, F., and Rabaud, M., 2005, "Decaying Grid-Generated Turbulence in a Rotating Tank," Phys. Fluids, 17(9), p. 095105.

[32] Staplehurst, P. J., Davidson, P. A., and Dalziel, S. B., 2008, "Structure Formation in Homogeneous, Freely Decaying, Rotating Turbulence," J. Fluid Mech., 598, pp. 81-103.

[33] Moisy, F., Morize, C., Rabaud, M., and Sommeria, J., 2011, "Decay Laws, Anisotropy and Cyclone-Anticyclone Anisotropy in Decaying Rotating Turbulence," J. Fluid Mech., 666, pp. 5-35.

[34] Davidson, P. A., Staplehurst, P. J., and Dalziel, S. B., 2006, "On the Evolution of Eddies in a Rapidly Rotating System," J. Fluid Mech., 557, pp. 135-144.

[35] Hopfinger, E., Browand, P., and Gagne, Y., 1982, "Turbulence and Waves in a Rotating Tank," J. Fluid Mech., 125, p. 505.

[36] Dickinson, S. C., and Long, R. R., 1983, "Oscillating-Grid Turbulence Including Effects of Rotation,” J. Fluid Mech., 126, pp. 315-333.

[37] Bokhoven, L. J. A. V., Clercx, H. J. H., Heijst, G. J. F. V., and Trieling, R. R. 2009, "Experiments on Rapidly Rotating Turbulent Flows," Phys. Fluids, 21(9), p. 096601 .

[38] Baroud, C. N., Plapp, B. B., She, Z.-S., and Swinney, H. L., 2002, "Anomalous Self-Similarity in a Turbulent Rapidly Rotating Fluid," Phys. Rev. Lett., 88, p. 114501.

[39] Del Castello, L., and Clercx, H. J. H., 2011, "Lagrangian Velocity Autocorrelations in Statistically Steady Rotating Turbulence,” Phys. Rev. E, 83(5), p. 056316.

[40] Lamriben, C., Cortet, P.-P., and Moisy, F., 2011, "Direct Measurements of Anisotropic Energy Transfers in a Rotating Turbulence Experiment," Phys. Rev. Lett., 107(2), p. 024503. 
[41] Kinzel, M., Holzner, M., Lüthi, B., Tropea, C., Kinzelbach, W., and Oberlack, M., 2009, "Experiments on the Spreading of Shear-Free Turbulence Under the Influence of Confinement and Rotation," Exp. Fluids, 47(4-5), pp. 801-809.

[42] Kinzel, M., Wolf, M., Holzner, M., Lüthi, B., Tropea, C., and Kinzelbach, W., 2010, "Simultaneous Two-Scale 3D-PTV Measurements in Turbulence Under the Influence of System Rotation," Exp. Fluids, 51(1), pp. 75-82.

[43] Ruppert-Felsot, J., Praud, O., Sharon, E., and Swinney, H., 2005, "Extraction of Coherent Structures in a Rotating Turbulent Flow Experiment," Phys. Rev. E, 72(1), p. 016311.

[44] Kolvin, I., Cohen, K., Vardi, Y., and Sharon, E., 2009, "Energy Transfer by Inertial Waves During the Buildup of Turbulence in a Rotating System," Phys. Rev. Lett., 102(1), p. 014503.

[45] Yarom, E., Vardi, Y., and Sharon, E., 2013, "Experimental Quantification of Inverse Energy Cascade in Deep Rotating Turbulence," Phys. Fluids, 25(8), p. 085105.

[46] Del Castello, L., and Clercx, H. J. H., 2011, "Lagrangian Acceleration of Passive Tracers in Statistically Steady Rotating Turbulence," Phys. Rev. Lett., 107(21), p. 214502.

[47] Del Castello, L., and Clercx, H. J., 2013, "Geometrical Statistics of the Vorticity Vector and the Strain Rate Tensor in Rotating Turbulence," J. Turbul, 14(10), pp. 19-36.

[48] Gallet, B., Campagne, A., Cortet, P.-P., and Moisy, F., 2014, "Scale-Dependent Cyclone-Anticyclone Asymmetry in a Forced Rotating Turbulence Experiment," Phys. Fluids, 26(3), p. 035108.

[49] Simand, C., Chillà, F., and Pinton, J.-F., 2000, "Inhomogeneous Turbulence in the Vicinity of a Large-Scale Coherent Vortex," Europhys. Lett. (EPL), 49(3) pp. 336-342.

[50] Lamriben, C., Cortet, P.-P., Moisy, F., and Maas, L. R. M., 2011, "Excitation of Inertial Modes in a Closed Grid Turbulence Experiment Under Rotation," Phys. Fluids, 23(1), p. 015102.

[51] Ferrero, E., Mortarini, L., Manfrin, M., Longhetto, A., Genovese, R., and Forza, R., 2009, "Boundary-Layer Stress Instabilities in Neutral, Rotating Turbulent Flows," Boundary Layer Meteorol., 130(3), pp. 347-363.

[52] Bewley, G. P., Lathrop, D. P., Maas, L. R. M., and Sreenivasan, K. R., 2007, "Inertial Waves in Rotating Grid Turbulence," Phys. Fluids, 19(7), p. 071701

[53] Ott, S., and Mann, J., 2000, "An Experimental Investigation of the Relative Diffusion of Particle Pairs in Three-Dimensional Turbulent Flow," J. Fluid Mech. 422, pp. 207-223.

[54] Morinishi, Y., Nakabayashi, K., and Ren, S., 2001, "A New DNS Algorithm for Rotating Homogeneous Decaying Turbulence,” Int. J. Heat Fluid Flow, 22(1), pp. $30-38$.

[55] Brandenburg, A., Svedin, A., and Vasil, G. M., 2009, "Turbulent Diffusion With Rotation or Magnetic Fields," Mon. Not. R. Astron. Soc., 395(3), pp. 1599-1606.

[56] Mininni, P., Rosenberg, D., and Pouquet, A., 2012, "Isotropisation at Small Scales of Rotating Helically Driven Turbulence," J. Fluid Mech., 699, pp. 263-279.

[57] Yeung, P. K., and Zhou, Y., 1998, "Numerical Study of Rotating Turbulence With External Forcing," Phys. Fluids, 10(11), pp. 2895-2909.

[58] Morinishi, Y., Nakabayashi, K., and Ren, S. Q., 2001, "Dynamics of Anisotropy on Decaying Homogeneous Turbulence Subjected to System Rotation," Phys. Fluids, 13(10), pp. 2912-2922.

[59] Thiele, M., and Müller, W.-C., 2009, "Structure and Decay of Rotating Homogeneous Turbulence," J. Fluid Mech., 637, pp. 425-442.

[60] Teitelbaum, T., and Mininni, P., 2010, "Large-Scale Effects on the Decay of Rotating Helical and Non-Helical Turbulence," Phys. Scr., 2010, p. 014003.
[61] Yoshimatsu, K., Midorikawa, M., and Kaneda, Y., 2011 "Columnar Eddy Formation in Freely Decaying Homogeneous Rotating Turbulence," J. Fluid Mech., 677, pp. 154-178.

[62] Delache, A., Cambon, C., and Godeferd, F., 2014, "Scale by Scale Anisotropy in Freely Decaying Rotating Turbulence," Phys. Fluids, 26(2), p. 025104.

[63] Deusebio, E., Boffetta, G., Lindborg, E., and Musacchio, S., 2014, "Dimensional Transition in Rotating Turbulence," Phys. Rev. E, 90, p. 023005.

[64] Dalziel, S. B., 2011, "The Twists and Turns of Rotating Turbulence," J. Fluid Mech., 666, pp. 1-4.

[65] Teitelbaum, T., and Mininni, P. D., 2011, "The Decay of Turbulence in Rotating Flows," Phys. Fluids, 23(6), p. 065105.

[66] Cambon, C., Mansour, N. N., and Godeferd, F. S., 1997, "Energy Transfer in Rotating Turbulence,” J. Fluid Mech., 337, pp. 303-332.

[67] Canuto, V. M., and Dubovikov, M. S., 1997, "A Dynamical Model for Turbulence. v. the Effect of Rotation,” Phys. Fluids, 9(7), pp. 2132-2140.

[68] Kraichnan, R. H., 1975, "Statistical Dynamics of Two-Dimensional Flow," J. Fluid Mech., 67(1), pp. 155-175.

[69] Bellet, F., Godeferd, F. S., Scott, J. F., and Cambon, C., 2006, "Wave Turbulence in Rapidly Rotating Flows," J. Fluid Mech., 562, pp. 83-121.

[70] Kassinos, S. C., Reynolds, W. C., and Roger, M. M., 2001, "One-Point Turbulence Structure Tensors,” J. Fluids. Mech., 428, pp. 213-248.

[71] Craya, A., 1958, "Contribution à l'analyse de la turbulence associée à des vitesses moyennes," Rev. Sci. et Tech. du Ministère de l'Air (France), $\mathbf{3 4 5}$

[72] Herring, J. R., 1974, "Approach of Axisymmetric Turbulence to Isotropy," Phys. Fluids, 17(5), pp. 859-872.

[73] Waleffe, F., 1993, "Inertial Transfers in the Helical Decomposition," Phys. Fluids A, 5(3), p. 026310.

[74] Bartello, P., Metais, O., and Lesieur, M., 1994, "Coherent Structures in Rotating 3-Dimensional Turbulence," J. Fluid Mech., 273, pp. 1-29.

[75] van Bokhoven, L., Cambon, C., Liechtenstein, L., Godeferd, F., and Clercx, H., 2008, "Refined Vorticity Statistics of Decaying Rotating Three-Dimensional Turbulence," J. Turbul., 9, N6.

[76] Gence, J. N., and Frick, C., 2001, "Birth of the Triple Correlations of Vorticity in an Homogeneous Turbulence Submitted to a Solid Body Rotation," C. R. Acad. Sci. Paris, Série IIB, 329(4), pp. 351-356.

[77] Sreenivasan, B., and Davidson, P. A., 2008, "On the Formation of Cyclones and Anticyclones in a Rotating Fluid," Phys. Fluids, 20(8), p. 085104.

[78] Zhou, Y., 1995, "A Phenomenological Treatment of Rotating Turbulence," Phys. Fluids, 7(8), pp. 2092-2094.

[79] Mahalov, A., and Zhou, Y., 1996, "Analytical and Phenomenological Studies of Rotating Turbulence," Phys. Fluids, 8(8), pp. 2138-2152.

[80] Galtier, S., 2003, "Weak Inertial-Wave Turbulence Theory," Phys. Rev. E, 68 p. 015301.

[81] Cambon, C., Rubinstein, R., and Godeferd, F. S., 2004, "Advances in WaveTurbulence: Rapidly Rotating Flows," New J. Phys., 6, 73.

[82] Galtier, S., 2014, "Theory for Helical Turbulence Under Fast Rotation," Phys. Rev. E, 89, p. 041001.

[83] Frisch, U., 1995, Turbulence. The Legacy of A. N. Kolmogorov, Cambridge University, Cambridge.

[84] Galtier, S., 2009, "Exact Vectorial Law for Homogeneous Rotating Turbulence," Phys. Rev. E, 80(4), p. 046301.

[85] Godeferd, F., and Cambon, C., 1994, "Detailed Investigation of Energy Transfers in Homogeneous Stratified Turbulence," Phys. Fluids, 6(6), pp. 2084-2100. 